\title{
Propuesta de modelo de gestión de activos fijos con enfoque en procesos ${ }^{*}$
}

\section{Asset management proposed model with focus on processes}

Recibido: 10 de abril de 2013

Revisado: 22 de mayo de 2013

Aceptado: 15 de agosto de 2013

Aida Ivonne Agudelo Pulido **

Convenio Universidad Santo Tomás e ICONTEC

\section{RESUMEN}

Los modelos de gestión facilitan a las organizaciones el logro de sus objetivos estratégicos desde diferentes facetas; cuando los activos fijos asociados a los procesos productivos son identificados por una organización como esenciales para el cumplimento de su misión, la gestión del mantenimiento y de su ciclo de vida revisten una gran importancia. Este artículo presenta una propuesta para un modelo integrado de gestión de activos fijos productivos con enfoque en procesos, basado en los requisitos de las normas técnicas ISO 9001:2008, ISO 55001:2014 y la guía PAS 55:2008 y sus relaciones, identificando los aspectos comunes, complementarios y específicos de estas normas técnicas, para lograr así un impacto en la disponibilidad y confiablidad de los activos fijos productivos y, en consecuencia, en la rentabilidad de las organizaciones.

* Este artículo corresponde a los resultados parciales de la investigación "Propuesta para integrar la gestión de la calidad y la gestión de activos fijos a partir de los modelos ISO 9001, ISO 55001 y PAS 55”, desarrollada para optar al título de magíster en Calidad y Gestión Integral.

** Correspondencia: Aida Ivonne Agudelo Pulido, ingeniera química, especialista en Ingeniería de Calidad y el Comportamiento, consultora en sistemas de gestión. Correo electrónico: aagudelp@hotmail.com. 
El modelo propuesto articula el sistema de gestión de calidad de la organización con el de gestión de sus activos fijos en forma coherente y práctica para su implementación simultánea, de manera que se evita la duplicidad de esfuerzos, se optimizan la disponibilidad y la confiabilidad de los activos fijos, y se suministran lineamientos que orientan sobre su entendimiento y aplicación.

Para el desarrollo del modelo se llevaron a cabo las etapas de diagnóstico, elaboración de la matriz de correlación, diagrama gráfico del modelo y la elaboración del documento de propuesta del modelo, el cual está organizado de acuerdo con la estructura de alto nivel del Anexo SL de la ISO.

Palabras clave: activos productivos, gestión de la calidad, ciclo de vida del activo, gestión de activos.

\section{ABSTRACT}

The management models enable organizations achieve their strategic objectives from different facets; when fixed assets associated with the production processes are identified by an organization as essential to the fulfillment of its mission, management of maintenance and life cycle are of great importance. This article presents a proposal for an integrated management system of productive fixed assets with focus on a process model, based on the requirements of technical standards ISO 9001:2008, ISO 55001:2014 and PAS Guide 55:2008 and their relations, which identifies common, complementary and specific aspects of these technical standards, in order to have an impact on the availability and reliability of productive fixed assets and consequently on the profitability of organizations.
The proposed model articulates the quality management system of the organization with the management of fixed assets in a consistent and practical way, for simultaneous implementation, avoiding duplication of efforts, optimizing the availability and reliability of fixed assets, and providing guidelines on their understanding and application.

To develop the model were conducted stages of diagnosis, development of the correlation matrix, graphical diagram of the model and the preparation of the proposal document model, which is organized according to the high-level structure of ISO's Annex SL.

Keywords: Oproductive assets, quality management, asset life cycle, assets management.n.

\section{INTRODUCCIÓN}

La implementación de un sistema de gestión permite a las organizaciones el análisis sistemático de su entorno y de los requerimientos de sus partes interesadas, con el fin de establecer objetivos y desarrollar la estructura organizacional, así como los documentos y los seguimientos necesarios para el logro de los dichos objetivos. En algunas organizaciones, los activos fijos son factores claves para el logro de dichos objetivos, para lo cual se requiere desarrollar una dinámica particular alrededor de la administración y el mantenimiento de estos activos, convirtiendo el ciclo de vida de los activos productivos en un proceso relevante para la operación.

La dinámica organizacional relacionada con la gestión de los activos productivos ha evolucionado a partir de los años con la filosofía que afirma que "la calidad de los mantenimientos también debe ser 
manejada sistemáticamente" ${ }^{1}$; en los años noventa se definió que la calidad "abarca todas las áreas de la organización y no solo a las que tienen contacto cara a cara con los clientes", lo cual es influenciado por la norma técnica ISO 9001:20002, ${ }^{3}$. En el año 2004 se estableció la PAS 554 como una guía para el aseguramiento de servicios en la generación y distribución de energía eléctrica en Gran Bretaña (actualizada en 2008), que proporciona elementos para alcanzar un mejor desempeño de los activos, asegurar la disponibilidad y confiabilidad de los equipos, administrar riesgos en los procesos, optimizar el gasto y actuar con responsabilidad respecto del medio ambiente, salud ocupacional y sociedad en general, manejando una relación costo-beneficio efectiva ${ }^{5}$. Y en enero de 2014 la Organización Internacional para la Normalización (ISO por sus siglas en inglés, International Organization for Standardiæation), por intermedio de su Comité 251, aprobó la serie de normas técnicas relacionadas con la gestión de

1 Los activos: la fiel representación financiera de los recursos empresariales [en línea]. En: Gestiópolis. [Citado en 19 de agosto de 2012]. Disponible en Internet: http://www.gestiopolis.com/canales/ financiera/articulos/no\%208/activos.htm p. 4.

2 AMENDOLA, Luis. Modelos mxtos en la gestión del mantenimiento [en línea]. PMM Institute For Learning. [Citado el 18 de agosto de 2012]. Disponible en Internet: http://www.pmmlearning. com/index.php?option=com_content\&view=article\&id=40\&Item $\mathrm{id}=27 \#$ p. 4 .

3 INSTITUTO COLOMBIANO DE NORMALIZACIÓN Y CERTIFICACIÓN. Sistemas de gestión de la calidad. Requisitos. NTC-ISO 9001. Bogotá, D.C.: El Instituto, 2008.

4 BRITISH STANDARDS INSTITUTION. (BSI) Asset Management. Publicly Available Specification PAS 55:2008, London: BSI, 2008. 76 p.

5 ISA mejora su gestión de activos [en línea]. Boletín 54. Junio 22 de 2011 [Citado el 18 de agosto de 2012]. Disponible en Internet: http://cliente.isa.com.co/index.php/editions/view/isa_mejora _su_gestion_de_activos activos ISO $55000^{6}$, que toma como referente inicial la PAS 55.

De acuerdo con esta información, las empresas certificadas con la norma técnica ISO 9001:2008, que manejan un enfoque por procesos y tienen como factor clave de éxito la gestión de activos, al integrar a su sistema de gestión de calidad -con las disposiciones de la Especificación British Standard, disponible al público para la gestión optimizada de activos físicos"7, guía PAS 55:2008-, incluyen las disposiciones aplicables al ciclo de vida del activo productivo para que la empresa asegure su disponibilidad, confiabilidad y la rentabilidad del negocio, evitando la duplicación de esfuerzos para el mejoramiento de su desempeño, traducido en términos de eficacia, eficiencia y efectividad de sus procesos y el logro de sus objetivos estratégicos.

A partir de esta necesidad se identifica la importancia de formular un modelo de gestión que integre los requisitos de un sistema de gestión de la calidad con base en la norma técnica ISO 9001:2008, con el sistema de gestión de activos productivos basado en la guía PAS 55:2008 y los requisitos de la norma técnica ISO 55001:2014.

Para la construcción de la propuesta del modelo integrado se tienen en consideración los siguientes documentos normativos:

6 BRITISH STANDARDS INSTITUTION. (BSI) PAS 55:2008. Gestión de activos Parte 1, ISBN: 978-0-9563934-0-1.

7 ¿Qué es PAS 55? [en línea]. PAS 55 Asset Management. [Citado el 29 de julio de 2012]. Disponible en Internet: http://pas55.net/ spanish/whatis.asp 
Tabla 1. Marco normativo

\begin{tabular}{|c|c|}
\hline Norma técnica & Breve descripción \\
\hline $\begin{array}{l}\text { NTC ISO 9000:2005. Sistemas de gestión } \\
\text { de la calidad. Fundamentos y vocabulario. }\end{array}$ & $\begin{array}{l}\text { Norma técnica internacional que describe los fundamentos y relaciona la terminología aplicable } \\
\text { a los sistemas de gestión de la calidad. }\end{array}$ \\
\hline $\begin{array}{l}\text { NTC-ISO 9001:2008. Sistemas de gestión } \\
\text { de la calidad. Requisitos. }\end{array}$ & $\begin{array}{l}\text { Norma técnica internacional que especifica los requisitos para un sistema de gestión de la } \\
\text { calidad que pueden utilizarse para su aplicación interna por las organizaciones, para certificación } \\
\text { o con fines contractuales. El objetivo se centra en la eficacia del sistema de gestión de la calidad } \\
\text { para satisfacer los requisitos del cliente. }\end{array}$ \\
\hline $\begin{array}{l}\text { Guía PAS 55-1:2008. Gestión de activos. } \\
\text { Parte 1. Especificaciones para la gestión } \\
\text { optimizada de activos físicos. }\end{array}$ & $\begin{array}{l}\text { Especificación británica disponible al público para la gestión de los activos de infraestructura } \\
\text { física. La primera parte corresponde a las especificaciones propiamente dichas; incluye } \\
\text { terminología y los requisitos específicos para el manejo de los activos durante su ciclo de vida. }\end{array}$ \\
\hline $\begin{array}{l}\text { Guía PAS 55-2:2008. Gestión de activos. } \\
\text { Parte 2. Directrices para la aplicación de la } \\
\text { guía PAS 55-1. }\end{array}$ & $\begin{array}{l}\text { Especificación británica disponible al público para la gestión de los activos de infraestructura } \\
\text { física. La segunda parte proporciona directrices para la aplicación de los requerimientos } \\
\text { especificados en la parte } 1 \text {, de tal forma que proporciona guías para el establecimiento del } \\
\text { sistema de gestión de activos y su coordinación con otros sistemas de gestión. }\end{array}$ \\
\hline Anexo SL ISO. & $\begin{array}{l}\text { Anexo que describe la estructura de alto nivel para la elaboración de normas técnicas de } \\
\text { sistemas de gestión, incluido en el documento que define los procedimientos básicos que se } \\
\text { deben seguir en el desarrollo de normas internacionales y otros estándares de la Organización } \\
\text { Internacional para la Estandarización (ISO). }\end{array}$ \\
\hline $\begin{array}{l}\text { Norma ISO 55001:2014. Gestión de activos. } \\
\text { Sistemas de gestión. Requisitos. }\end{array}$ & $\begin{array}{l}\text { Norma técnica internacional ISO, primera edición, que especifica los requisitos para el } \\
\text { establecimiento, la implementación, el mantenimiento y la mejora de un sistema para la gestión } \\
\text { de activos, conocido como un sistema de gestión de activos. }\end{array}$ \\
\hline
\end{tabular}

Fuente: autora

NTC ISO 9000:2005 ${ }^{8}$, NTC-ISO 9001:20089 , guía PAS 55-1:2008", guía PAS 55-2:2008¹, anexo SL ISO ${ }^{12}$, norma ISO 55001:2014 ${ }^{13}$.

8 INSTITUTO COLOMBIANO DE NORMALIZACIÓN Y CERTIFICACIÓN. Sistemas de gestión de la calidad. Fundamentos y vocabulario. NTC-ISO 9000. Bogotá, D.C.: El Instituto, 2000.36 p.

9 INSTITUTO COLOMBIANO DE NORMALIZACIÓN Y CERTIFICACIÓN. Sistemas de gestión de la calidad. Requisitos. NTC-ISO 9001. Bogotá, D.C.: El Instituto, 2008.

10 BRITISH STANDARDS INSTITUTION. Gestión de activos. Guía PAS 55-1:2008. p.1.

11 BRITISH STANDARDS INSTITUTION. Gestión de activos. Guía PAS 55-2:2008. Londres: UK, 2008. ISBN: 978099563934-2-5: BSI.

12 INTERNATIONAL ORGANIZATION FOR STANDARDIZATION / INTERNATIONAL ELECTROTECHNICAL COMMISSION. Directives. Part 1. Consolidated ISO Supplement Procedures Specific to ISO. Ginebra, Suiza: ISO, 2013. p 62.

13 INTERNATIONAL ORGANIZATION FOR STANDARDIZATION. Gestión de activos. istemas de gestión. Requisitos. ISO $55001: 2014$. Ginebra, Suiza: ISO, 2014. 


\section{METODOLOGÍA}

La propuesta del modelo se realiza partiendo de lo particular a lo general, mediante el estudio, el análisis y la correlación de los documentos técnicos normativos anteriormente citados.

La propuesta del modelo puede ser utilizada por cualquier tipo de empresa que identifique la gestión de activos como un factor clave de éxito en su planeación estratégica, con el fin de asegurar una mayor disponibilidad y confiabilidad de los activos y la rentabilidad del negocio en el desarrollo de los procesos operativos de la organización.

Para alcanzar el objetivo de definir un modelo de gestión que permita integrar el enfoque de procesos establecido en la norma técnica ISO 9001:2008 y la gestión de activos productivos fundamentada en el estándar guía PAS 55:2008, se realizan el estudio y el análisis de la información inicial de estas dos normas técnicas, se toman en cuenta la revisión adicional y el análisis de los requisitos de la recientemente aprobada ISO 55001:2014. Posteriormente los requisitos se integran a partir de sus propósitos, con el fin de identificar elementos comunes tanto en la concepción de los requisitos como en la información documentada requerida.

La formulación de la propuesta del modelo incluye las siguientes etapas: diagnóstico, elaboración de la matriz de correlación, diagrama gráfico del modelo y elaboración del modelo.

En la etapa de diagnóstico se realiza un estudio de los antecedentes del enfoque de procesos y de la gestión de activos. Se revisa la información relacionada con la planeación estratégica y la documentación del sistema de gestión de la calidad de una empresa del sector eléctrico colombiano certificada con ISO 9001:2008. Este diagnóstico permite concluir que el modelo integrado es necesario en aquellas organizaciones que identifiquen a los activos productivos como factores claves de éxito para el cumplimiento de su misión.

La elaboración de la matriz de correlación parte de la identificación de los requisitos comunes, complementarios y diferentes -organizados de acuerdo con el ciclo PHVA- ${ }^{-14}$ de las normas técnicas ISO 9001 y la guía PAS 55, inicialmente, adicionando luego la norma ISO 55001. El análisis incluye la revisión e interpretación de los requisitos de los referenciales por parte del investigador, de acuerdo con su experticia, para identificar su correlación y organizar la matriz, considerando que los requisitos se asocian a los procesos que las organizaciones identifican y corresponden a un ciclo de gestión PHVA.

Posteriormente se revisa la estructura de alto desempeño del anexo SL de la ISO, con el propósito de que la propuesta del modelo de gestión cumpla dicha estructura planteada para las normas técnicas $\mathrm{ISO}^{15}$, de manera que luego se pueda articular con otros modelos de gestión. De tal forma que la matriz de correlación generada incluye los requisitos de las normas técnicas ISO 9001:2008, ISO 55001:2014 y la guía PAS 55:2008, y se estructura con base en el documento anexo SL de la ISO.

Para la actualización de la matriz de correlación con base en la tabla comparativa organizada de acuerdo

14 El ciclo de gestión PHVA se puede describir así: Planificar, es decir, establecer los objetivos y procesos necesarios para conseguir resultados de acuerdo con los requisitos del cliente y las políticas de la organización; Hacer, esto es, implementar los procesos; Verificar, lo que implica realizar el seguimiento y la medición de los procesos y los productos respecto a las políticas, los objetivos y los requisitos para el producto e informar sobre los resultados; y Actuar, en otras palabras, tomar acciones para mejorar continuamente el desempeño de los procesos.

15 INTERNATIONAL ORGANIZATION FOR STANDARDIZATION/INTERNATIONAL ELECTROTECHNICAL COMMISSION. Directives, Part 1. Consolidated ISO Supplement Procedures Specific to ISO. Op. cit., p. 62. 
con la estructura del documento anexo SL, las normas técnicas ISO 9001:2008 e ISO 55001:2014 y la guía PAS 55:2008, el ejercicio metodológico que se realiza es el siguiente: se revisan los componentes de estructura general del anexo SL, para identificar los elementos y requisitos relacionados, con base en los cuales se encuentra la norma técnica ISO 55001. Posteriormente se verifican los requisitos de la norma técnica ISO 55001:2014 con respecto a los requisitos de la guía PAS 55:2008, para identificar los ajustes en el orden de la ubicación de dichos requisitos en la matriz de correlación y, por lo tanto, en los numerales asignados.

Se revisa que los numerales de la norma técnica ISO 9001:2008, alineados con los numerales de la guía PAS 55:2008, se encuentren ubicados en el componente correspondiente de acuerdo con el anexo SL ISO. Al realizar esta revisión se encuentra que algunos numerales deben ser desplazados, lo que implica cambiar su orden a fin de que coincidan con la estructura del anexo SL.

Algunos de los cambios más representativos identificados en la definición inicial del modelo de acuerdo con la estructura del anexo SL y de la norma técnica ISO 55001:2014 son los siguientes:

- La gestión documental (numeral 4.2 en la norma técnica ISO 9001:2008) no corresponde al componente de contexto estratégico se encuentran las generalidades del sistema de gestión, sino al componente de soporte.

- El requisito de enfoque al cliente, que anteriormente formaba parte del capítulo de responsabilidad de la dirección (capítulo 5 en la norma técnica ISO 9001:2008), pasa del liderazgo al componente de contexto estratégico.

- Los requisitos de identificación y revisión de requisitos (numerales 7.2.1 y 7.2.2 en la norma técnica ISO 9001:2008) pasan de la parte operativa a la de contexto estratégico.

- La revisión por la dirección (numeral 5.6 en la norma técnica ISO 9001:2008) pasa del componente de liderazgo al de evaluación del desempeño.

- Se ajusta la matriz de correlación para que los numerales queden ubicados en el componente al que corresponden.

- De acuerdo con el cambio de los numerales descritos anteriormente, se hace necesario ajustar la columna donde se especifica la etapa del PHVA a la que correspondía cada uno de los requisitos.

- Como resultado, también se revisa la diagramación inicial de articulación del modelo.

- Como uno de los aspectos claves para la gestión de activos productivos, se incluye el tema de manejo del riesgo identificado en el punto 4.4.7 de la guía PAS 55:2008, en la norma técnica ISO 55001:2014 en el numeral 6.1 dentro del capítulo de Planificación y en el anexo SL, que establece acciones para tratar riesgos y oportunidades. De acuerdo con estos estándares, la organización debe identificar los riesgos y oportunidades, es decir, los efectos positivos y negativos que puedan presentarse, e incidir en los resultados de la gestión de la calidad y de los activos fijos.

De tal forma, la matriz permite la identificación de los requisitos comunes, complementarios y particulares de la normatividad correspondiente a los modelos de gestión de la calidad y activos, y responde al orden de la estructura del anexo SL y el ciclo PHVA de la organización, identificando el tipo de proceso típico en el cual se refleja el requisito, tales como estratégico, misional y de apoyo. 


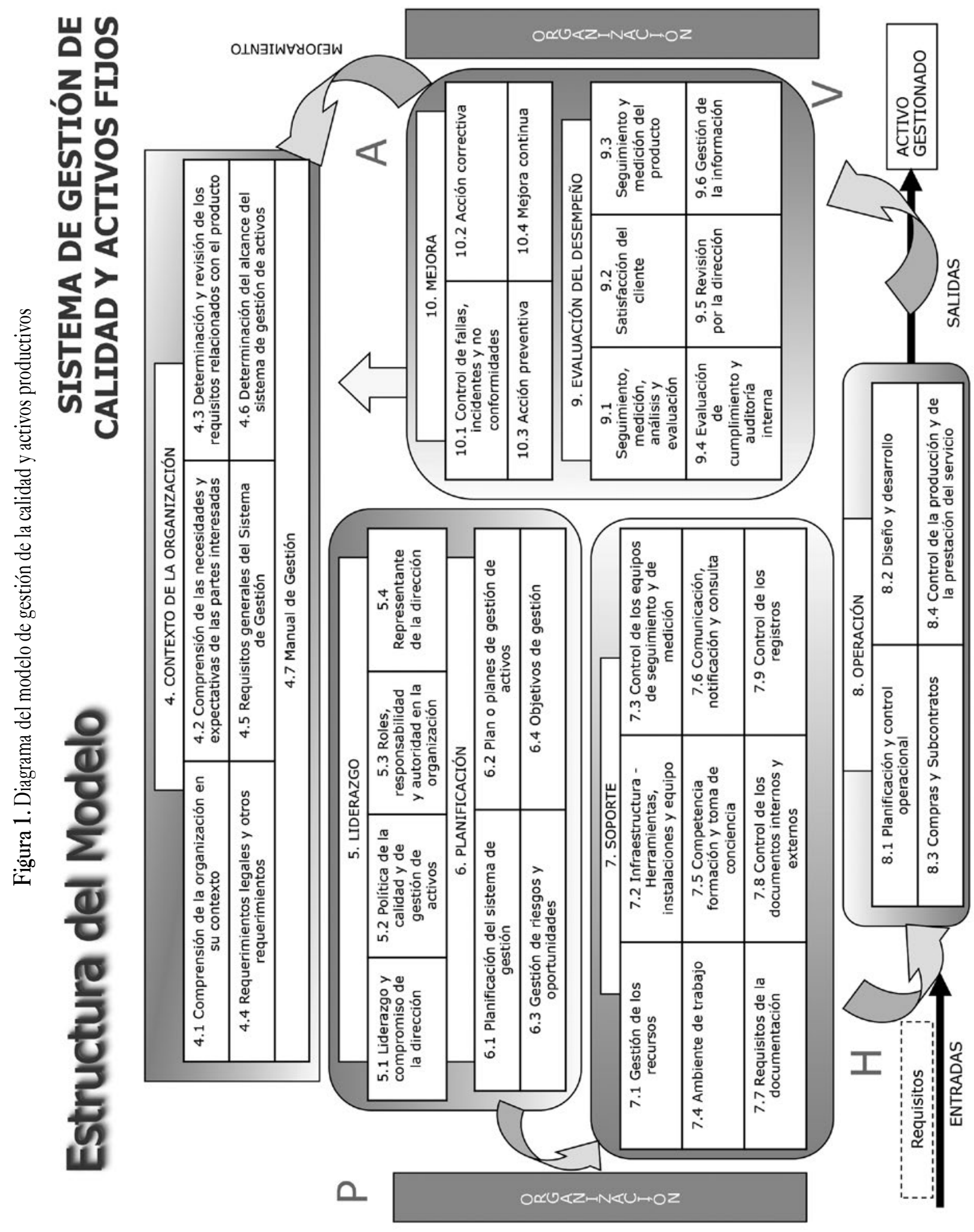


La figura 1 presenta el diagrama gráfico de la propuesta del modelo que se elabora a partir de la diagramación individual de cada una de las normas técnicas objeto de estudio y mencionadas en la tabla 1 . De tal forma que el diagrama representa de forma gráfica los requisitos comunes, complementarios y particulares organizados de acuerdo con la estructura del anexo SL, y con una nominación que da la investigadora para cada uno de los requisitos del modelo de gestión de la calidad y activos fijos propuesto.

El documento final de propuesta del modelo se estructuró de acuerdo con las políticas definidas para la elaboración de normas técnicas de sistemas de gestión ${ }^{16}$ (anexo SL) y presenta la siguiente tabla de contenido:

- Introducción

- 0.1 Generalidades

- 0.2 Enfoque basado en procesos

- $\quad 0.3$ Tipo de activos

- 0.4 Compatibilidad con otros sistemas de gestión

- 1. Objeto y campo de aplicación

- 1.1 Generalidades

- 1.2 Aplicación y alcance

- 2. Referencias normativas

- 2.1 Publicaciones de referencia

16 INTERNATIONAL ORGANIZATION FOR STANDARDIZATION/INTERNATIONAL ELECTROTECHNICAL COMMISSION. Directives, Part 1. Consolidated ISO Supplement Procedures Specific to ISO. Op. cit., p. 62.
- 3. Términos y definiciones

- 4. Contexto de la organización

- 5. Liderazgo

- 6. Planificación

- 7. Soporte

- 8. Operación

- 9. Evaluación del desempeño

- 10. Mejora

Los capítulos correspondientes a los requisitos del modelo de gestión de la calidad y la gestión de activos fijos corresponden a los numerales 4 a 10.

La propuesta del modelo incluye para cada uno de los requisitos una tabla (tabla 2.) con lineamientos para la integración de los referenciales en el modelo de gestión de la calidad y de activos, que se observa más adelante:

De esta forma, la propuesta del modelo tiene la estructura y contenido de una norma técnica internacional, incluye el modelo gráfico que lo describe y una tabla resumen de la alineación de los requisitos de los referenciales tomados como base para su construcción con los lineamientos que facilitan su implementación. 


\section{RESULTADOS}

De las etapas de la investigación y la construcción de la propuesta del modelo se obtienen los siguientes resultados:

La identificación de los activos productivos como un factor clave de éxito y rentabilidad del negocio resultante del diagnóstico.

La matriz teórica elaborada con las normas técnicas ISO 9001:2008, ISO 55001:2014 y la guía PAS 55:2008 permite identificar los requisitos comunes, complementarios y particulares para el modelo integrado al reconocer como aspectos comunes los siguientes requisitos: control de documentos y registros, recurso humano, provisión de recursos, infraestructura, control de dispositivos de seguimiento y medición, seguimiento y medición, acciones correctivas y revisión por la dirección, de tal forma que el modelo permite integrar estos requisitos y sus disposiciones para la gestión.

La matriz teórica de la correlación de las normas técnicas ISO 9001:2008, ISO 55001:2014 y la guía PAS 55:2008, tomando como referencia la estructura del anexo SL de la ISO, así como la identificación del tipo de actividad dentro de un ciclo de gestión PHVA acorde con el diagrama del modelo, permite definir el propósito del requisito conforme con los principios y enfoques de los referenciales, el lineamiento o consideraciones que posibilitan la alineación e integración entre los modelos, así como la identificación del tipo de procesos en los que se aplican, lo cual se encuentra definido en el tabla guía de requisitos del modelo propuesto.

El modelo propuesto, que integra los requisitos de las normas técnicas ISO 9001:2008, ISO 55001:2014 y la guía PAS 55:2008, se presenta como un documento estructurado de acuerdo con las políticas definidas para elaboración de normas técnicas de sistemas de gestión ${ }^{17}$ (anexo SL). Este documento incluye, para cada uno de los requisitos, un tabla de lineamientos para la integración de los referenciales en el modelo de gestión de la calidad y activos fijos, en la que se especifican el capítulo del anexo SL al que corresponde el requisito, el nombre y el numeral del requisito en el modelo, el requisito de la norma técnica ISO 9001:2008 relacionado, el requisito de la guía PAS 55:2008 respectivo, el requisito de la norma técnica ISO 55001:2014 asociado, los comentarios al requisito de la norma técnica ISO 55001:2014 con respecto al requisito relacionado de la guía PAS 55:2008, la etapa del PHVA del sistema de gestión a la que corresponde el requisito, el propósito común de los requisitos identificados como relacionados, las orientaciones y los lineamientos sobre particularidades que son aplicables a los dos sistemas de gestión y que es recomendable articular y trabajar de manera unificada, y el tipo de proceso que da aplicación al requisito del modelo (estratégico, misional, de apoyo o de evaluación).

El análisis comparativo de los modelos normalizados estudiados permite determinar la ciudadanía sistémica del modelo e identificar la ubicación de los requisitos comunes y las disposiciones aplicables, siendo la ciudadanía sistémica los procesos, debido a que en estos convergen los requisitos y disposiciones aplicables. De otra parte, las cualidades emergentes corresponden a las características o requisitos del modelo de gestión que hace posible integrar el enfoque de procesos y la gestión de activos productivos sin perder la identidad de cada uno de los sistemas de gestión que lo conforman, calidad y gestión de activos en consonancia con los objetivos del sistema integrado de la organización.

17 INTERNATIONAL ORGANIZATION FOR STANDARDIZATION/INTERNATIONAL ELECTROTECHNICAL COMMISSION. Directives, Part 1. Consolidated ISO Supplement Procedures Specific to ISO, Op. cit., p. 62. 
Tabla 2. Tabla guía del modelo de gestión de la calidad y de activos fijos

\begin{tabular}{|c|c|c|c|}
\hline Anexo SL - ISO & \multicolumn{3}{|c|}{ Corresponde al capítulo del anexo SL para el requisito. } \\
\hline $\begin{array}{l}\text { Requisito } \\
\text { del modelo }\end{array}$ & \multicolumn{3}{|c|}{ Requisito del modelo. Denominación dada por la investigadora. } \\
\hline ISO 9001:2008 & \multicolumn{3}{|c|}{ Requisito de la norma técnica ISO 9001:2008 relacionado con el requisito del modelo. } \\
\hline BSI PAS 55:2008 & \multicolumn{3}{|c|}{ Requisito de la guía PAS 55:2008 relacionado con el requisito del modelo. } \\
\hline ISO 55001:2014 & $\begin{array}{l}\text { Requisito de la norma técnica } \\
\text { ISO 55001:2014 relacionado con } \\
\text { el requisito del modelo. }\end{array}$ & Comentarios versión 2014 & $\begin{array}{l}\text { Comentarios al requisito de la } \\
\text { norma técnica ISO 55001:2014 } \\
\text { con relación al requisito } \\
\text { relacionado de la guía PAS } \\
\text { 55:2008. }\end{array}$ \\
\hline Orientación PHVA & \multicolumn{3}{|c|}{$\begin{array}{l}\text { Etapa del PHVA del sistema de gestión a la que corresponde el requisito específico del modelo y los requisitos } \\
\text { asociados de la norma técnica ISO 9001:2008, la guía PAS 55:2008 y la norma técnica ISO 55001:2014. }\end{array}$} \\
\hline $\begin{array}{l}\text { Propósito } \\
\text { del requisito }\end{array}$ & \multicolumn{3}{|c|}{ Propósito común de los requisitos identificados como relacionados. } \\
\hline $\begin{array}{l}\text { Lineamiento para } \\
\text { la integración del modelo }\end{array}$ & \multicolumn{3}{|c|}{$\begin{array}{l}\text { Orientaciones y lineamientos sobre particularidades que son aplicables a los dos sistemas de gestión y que se } \\
\text { recomienda articular y trabajar de manera unificada. }\end{array}$} \\
\hline Tipo de proceso & \multicolumn{3}{|c|}{$\begin{array}{l}\text { Tipo de proceso que da aplicación al requisito del modelo. Estos tipos de proceso pueden ser estratégico, } \\
\text { misional, de apoyo o de evaluación. }\end{array}$} \\
\hline
\end{tabular}

Fuente: autora

\section{CONCLUSIONES}

La propuesta del modelo de gestión de la calidad y activos fijos, que define la articulación entre los aspectos de planeación, desempeño y seguimiento durante el ciclo de vida de los activos en la realización de las actividades de la empresa, acordes con el plan estratégico, favorece el cumplimiento de los indicadores de crecimiento establecidos y rentabilidad esperada.

De acuerdo con el diagnóstico, se identifica que los activos productivos son un factor clave de éxito y rentabilidad del negocio, de tal forma que la propuesta del modelo de gestión de la calidad y activos fijos, que permite integrar el enfoque de procesos y la gestión de activos productivos, contribuye al logro de los objetivos estratégicos de las organizaciones en las cuales son relevantes para el cumplimiento de su misión los activos fijos.

La elaboración de la matriz de correlación evidencia que los aspectos identificados como relevantes para la articulación entre un sistema de gestión de la calidad certificado de acuerdo con los requisitos de la norma técnica ISO 9001:2008 y la gestión de activos productivos basada en la guía PAS 55:2008, así como la ISO 55001 son el enfoque de procesos y la administración de los activos productivos en todos los momentos de su ciclo de vida.

A partir de la matriz teórica de correlación de los referenciales y su ubicación dentro del modelo de sistema de gestión estructurado de acuerdo con el anexo SL, se establecen las disposiciones y los aspectos comunes, 
de tal manera que los requisitos y disposiciones aplicables del sistema de gestión de la calidad basado en los requisitos de la norma técnica ISO 9001 en su actividad específica de prestación de servicios, se integran de manera práctica y eficaz con la gestión de activos productivos basada en la norma técnica ISO 55001 y la guía PAS 55, reduciendo así la duplicidad de esfuerzos en la gestión de sus procesos y el cumplimiento de los diferentes referenciales.

La construcción del diagrama y de la matriz de correlación teórica, las normas técnicas ISO 9001:2008, ISO 55001:2014 y la guía PAS 55:2008, de acuerdo con el enfoque PHVA y la estructura del anexo SL de la ISO, permite desarrollar una propuesta de modelo de gestión acorde con estas normas técnicas internacionales, de fácil comprensión por parte de los líderes o responsables de los procesos de cualquier organización encargados de su implementación y mantenimiento, que puede usarse como base para la integración de otros modelos de gestión.

El modelo propuesto incluye el tema de manejo del riesgo de acuerdo con lo establecido en el anexo SL, que establece acciones para tratar riesgos y oportunidades y lo identificado en el punto 4.4.7 de la guía PAS 55:2008, en la norma técnica ISO 55001:2014, numeral 6.1, capítulo de Planificación, así como también en la norma técnica ISO 9001:2008, apartado 8.5.3 que trata de acciones preventivas. Estos estándares establecen que la organización debe identificar los riesgos y las oportunidades, es decir, efectos positivos y negativos que puedan presentarse e incidir en los resultados de la gestión de la calidad y de los activos fijos.

\section{RECOMENDACIONES}

Para la implementación del modelo se recomienda partir de la planeación estratégica y del enfoque de procesos, considerando el factor clave de éxito de los activos en la actividad de la organización.

Se sugiere modelar los procesos de la empresa con base en el ciclo de vida de los activos y la misión de la organización, garantizando que por medio de los procesos identificados sea posible dar cumplimiento a los objetivos estratégicos de la organización.

Conviene realizar un análisis detallado de la relación entre los procesos de la organización y los requisitos del modelo, toda vez que este propone el proceso típico. Asimismo se recomienda que en el momento de la implementación del modelo se revise a qué proceso corresponde la administración del cumplimiento de cada uno de los requisitos mencionados, para lo cual se debe considerar el lineamiento propuesto y el producto o evidencia que demostrará dicho cumplimiento.

Para integrar los requisitos de los estándares relacionados de calidad y activos de acuerdo con el modelo propuesto, se sugiere iniciar por requisitos de los comunes, y de lo general a lo particular, apoyándose en los lineamientos definidos en el modelo propuesto.

Como continuidad del proyecto de investigación, el cual considera tres referenciales y dos modelos de gestión (el de calidad ISO 9001 y los de gestión de activos productivos guía PAS 55 y norma técnica ISO 55001:2014), se puede ampliar luego a otros sistemas de gestión, incluyendo la norma de gestión de riesgos ISO 31000, un sistema de gestión ambiental ISO 14001 y un sistema de seguridad y salud en el trabajo OHSAS 18001.

Se hace necesario hacer un pilotaje de la propuesta del modelo para su validación y la de su aplicación, para lo cual se sugiere una empresa perteneciente al sector de generación de energía eléctrica. 


\section{REFERENCIAS}

AMENDOLA, Luis. Modelos mixtos en la gestión del mantenimiento [en línea]. PMM InstituteForLearning. [Citado el 18 de agosto de 2012]. Disponible en Internet: http://www.pmmlearning.com/ index.php?option=com_content\&view=article\&i $\mathrm{d}=40 \&$ Itemid $=27 \# 6 \mathrm{p}$.

BRITISH STANDARDS INSTITUTION (BSI). Gestión de activos. Parte 1, PAS 55:2008. ISBN: 978-0-9563934-0-1.

BRITISH STANDARDS INSTITUTION. (BSI), Gestión de Activos Parte 2, PAS 55:2008. ISBN: 978-0-9563934-2-5.

ESCOBAR PÉREZ, Jazmine y CUERVO MARTÍNEZ, Ángela. Validez de contenido y juicio de expertos: una aproximación a su utilización. En: Avances en Medición. Abril, 2008, n. ${ }^{0}$ 6, p. 27-36 [en línea]. Consultado el 23 de abril de 2014. Disponible en internet: http://www.humanas.unal. edu.co/psicometria/files/7113/8574/5708/Articu103_Juicio_de_expertos_27-36.pdf. 10 p.

INSTITUTO COLOMBIANO DE NORMALIZACIÓN Y CERTIFICACIÓN. Sistemas de gestión de la calidad. Fundamentos y vocabulario. NTCISO 9000. Bogotá, D.C.: El instituto, 2000. 36 p.

INSTITUTO COLOMBIANO DE NORMALIZACIÓN Y CERTIFICACIÓN. Sistemas de gestión de la calidad. Requisitos. NTC-ISO 9001. Bogotá, D.C.: El Instituto, 2008. 35 p.

INTERNATIONAL ORGANIZATION FOR STANDARDIZATION/INTERNATIONAL
ELEGTROTECHNICAL COMMISSION. Directives, Part 1. Consolidated ISO Supplement Procedures Specific to ISO. Ginebra, Suiza: ISO, 2013. 62 p.

INTERNATIONAL ORGANIZATION FOR STANDARDIZATION. Gestión de activos. Sistemas de gestión. Requisitos. ISO 55001:2014. Ginebra, Suiza: ISO, 2014.

ISA mejora su gestión de activos [en línea]. Boletín 54. Junio 22 de 2011. [Citado el 18 de agosto de 2012]. Disponible en Internet: http:// cliente.isa.com.co/index.php/editions/view/ isa_mejora_su_gestion_de_activos

Los activos: la fiel representación financiera de los recursos empresariales [en línea]. En: Gestiópolis [Citado el 19 de agosto de 2012]. Disponible en Internet: http://www.gestiopolis.com/canales/ financiera/articulos/no\%208/activos.htm

¿Qué es PAS 55? [en línea]. PAS 55 Asset Managment [Citado el 29 de julio de 2012].. Disponible en Internet: http://pas55.net/spanish/whatis.asp

QUINTERO, José Eduardo. Gestión de activos en empresas de distribución de energía eléctrica. En: El observador de los Servicios Públicos. Septiembre, 2010, vol. 1, n. ${ }^{0}$ 5. p. 19.

ZULUAGA GIRALDO, Ramiro Antonio. Creación y consolidación de empresas. [ebrary]. Colombia: Ecoe Ediciones, 2007. p 306. [Citado el 19 de agosto de 2012]. Disponible en Internet: http://site.ebrary.com/lib/bibliotecaustasp/ Doc?id=10584162\&ppg=330 